\title{
Commutators of Foliation Preserving Homeomorphisms for Certain Compact Foliations
}

\author{
Dedicated to Professor Hiroyasu Ishimoto on his 60th birthday
}

$\mathrm{By}$

Kazuhiko FUKU1*

\section{§1. Introduction}

Let $M$ be an $u$-dimensional closed topological manifold. By $\mathscr{H}(M)$ we denote the group of all homeomorphisms of $M$ which are isotopic to the identity by an isotopy fixed outside a compact set.

In this note we treat certain subgroups of $\mathscr{H}(M)$.

Let $(M, N)$ be a manifold pair, where $N$ is a proper submanifold of $M$. Let $\mathscr{H}(M, N)$ denote the subgroup of homeomorphisms of $\mathscr{H}(M)$ which are invariant on $N$.

In $\S 2$, we consider the homologies of $\mathscr{H}(M, N)$, that is, the homology groups of the group $\mathscr{H}(M, N)$ and show that the homologies of $\mathscr{H}\left(\mathbb{R}^{n}, \mathbb{R}^{p}\right) \quad(p>0)$ vanish in all dimension $>0$. This is a special case of a result of Fukui-Imanishi[F-I] which is a generalization of a result of Mather $[\mathrm{Ma}]$ to foliated manifolds. We show in $\S 3$ that $\mathscr{H}(M, N)$ is perfect, i.e.. is equal to its own commutator subgroup, for a certain manifold pair $(M, N)$.

In $\S 4$ and $\S 5$, we consider the group of foliation preserving homeomorphisms. We have already discussed in $[\mathrm{F}-\mathrm{I}]$ about the case of codimension one foliations. We study here the case of compact foliations of codimension greater than one. Let $(M, \mathscr{F})$ be a $C^{1}$-foliated manifold and $F(M, \mathscr{F})$ be the group of foliation preserving homeomorphisms of $(M, \mathscr{F})$ isotopic to the identity by a foliation preserving isotopy fixed outside a compact

Communicated by K. Saito. April 30, 1997.

1991 Mathematics Subject Classıfication (s) : 57R30

* Department of Mathematics, Kyoto Sangyo University, Kyoto 603-8555, Japan.

e-mail: fukui @i,cc.kyoto-su.ac.jp 
set. Using the results in $\S 3$ we show in $\S 4$ that $F(M, \mathscr{F})$ is perfect for the case that $\mathscr{F}$ is a compact codimension two foliation with no dihedral leaves on a compact manifold $M$ and show in $\S 5$ that $F(M, \mathscr{F})$ is perfect for the case that $\mathscr{F}$ is a certain compact Hausdorff codimension three foliation with $\pi_{1}(L) \cong \mathbb{Z}$ for each leaf $L$ on a compact manifold $M$.

\section{§2. Homologies of $\mathscr{H}\left(\mathbb{R}^{n}, \mathbb{R}^{p}\right)$}

We recall that if $G$ is any group, then there is a standard chain complex $C(G)$ whose homology is the homology of $G$.

Let $C_{r}(G)$ be the free abelian group on the set of all $r$-tuples $\left(g_{1}, \ldots, g_{r}\right)$, where $g_{\imath} \in G$. The boundary operator $\partial: C_{r}(G) \rightarrow C_{r-1}(G)$ is defined by

$$
\partial\left(g_{1}, \ldots, g_{r}\right)=\left(g_{1}^{-1} g_{2}, \ldots, g_{1}^{-1} g_{r}\right)+\sum_{i=1}^{r}(-1)^{\imath}\left(g_{1}, \ldots, \hat{g}_{1}, \ldots, g_{r}\right) .
$$

Then we have $\partial^{2}=0$. The symbol $H_{r}(G)$ will stand for the $r$-th homology group of the above chain complex.

Let $\mathbb{R}^{n}=\left\{\left(x_{1}, \ldots, x_{n}\right) \mid x_{i} \in \mathbb{R}\right\}$ be an $n$-dimensional Euclidean space and $\mathbb{R}^{p}$ the $p$-dimensional subspace $\left\{\left(x_{1}, \ldots, x_{p}, 0, \ldots, 0\right) \mid x_{i} \in \mathbb{R}\right\}$ of $\mathbb{R}^{n}$.

Let $U$ is an open rectangle in $\mathbb{R}^{n}$ such that $U \cap \mathbb{R}^{p} \neq \varnothing$. We put $\mathscr{H}_{U}\left(\mathbb{R}^{n}, \mathbb{R}^{p}\right)=\left\{\left.f \in \mathscr{H}\left(\mathbb{R}^{n}, \mathbb{R}^{p}\right)\right|_{\text {supp }}(f) \subset U\right\}$. Let $\iota: \mathscr{H}_{U}\left(\mathbb{R}^{n}, \mathbb{R}^{p}\right) \rightarrow \mathscr{H}\left(\mathbb{R}^{n}, \mathbb{R}^{p}\right)$ denote the inclusion map, and let $\iota_{*}: H_{r}\left(\mathscr{H}_{U}\left(\mathbb{R}^{n}, \mathbb{R}^{p}\right)\right) \rightarrow H_{r}\left(\mathscr{H}\left(\mathbb{R}^{n}, \mathbb{R}^{p}\right)\right)$ denote the induced homomorphism. By the similar argument as in the proof of Lemma 2.2 of $[\mathrm{F}-\mathrm{I}]$, we have the following lemma.

Lemma 2.1. $\quad$ * is an isomorphism.

Theorem 2.2. If $p>0$, then the homology groups $H_{r}\left(\mathscr{H}\left(\mathbb{R}^{n}, \mathbb{R}^{p}\right)\right)=0$ for $r>0$.

Proof. We put $U=(1,2) \times(-1,1)^{n-1} \subset \mathbb{R}^{n}$. Then we note $U \cap \mathbb{R}^{p}=(1,2)$ $\times(-1,1)^{p-1}$. Take a homeomorphism $\phi \in \mathscr{H}\left(\mathbb{R}^{n}, \mathbb{R}^{p}\right)$ given by $\phi(x)=\frac{1}{3} x$ for $x$ $\in B(0,3)=\left\{x=\left(x_{1}, \ldots, x_{n}\right) \in \mathbb{R}^{n} \mid\left(x_{1}\right)^{2}+\cdots+\left(x_{n}\right)^{2}<9\right\}$. We set $U_{j}=\phi^{j}(U)=\left(\frac{1}{3^{\prime}}, \frac{2}{3^{\prime}}\right)$ $\times\left(-\frac{1}{3^{\prime}}, \frac{1}{3^{\prime}}\right)^{n-1},(j=0,1,2, \ldots)$. Note that $U_{0}=U$.

Then we have that $\bar{U}_{j} \cap \bar{U}_{k}=\emptyset$ if $j \neq k$ and $\left\{\bar{U}_{j}\right\}$ shrinks to the origin $0 \in$ $\mathbb{R}^{n}$ as $j$ goes to $\infty$.

The rest is proved by the similar way as in the proof of Theorem 2.1 of $[\mathrm{F}-\mathrm{I}]$.

Corollary 2.3. $\quad \mathscr{H}_{U}\left(\mathbb{R}^{n}, \mathbb{R}^{p}\right)$ and $\mathscr{H}\left(\mathbb{R}^{n}, \mathbb{R}^{p}\right)$ are perfect groups for $p>0$.

Proof. These are immediate consequences of Theorem 2.2 because that 
$H_{1}(G) \cong G /[G, G]$ for any group $G$.

\section{§3. Commutators of $\mathscr{H}(M, N)$}

A locally flat proper manifold pair $(M, N)$ is by definition a pair of topological manifolds such that $N$ is a locally flat submanifold of $M$, properly imbedded as a closed subset.

In this section, we show that $\mathscr{H}(M, N)$ is perfect for a locally flat manifold pair $(M, N)(\operatorname{dim} N \geq 0)$. For $\operatorname{dim} N>0$, we have the following as a corollary of Corollary 2.3.

Theorem 3.1. Let $(M, N)$ be a locally flat proper manifold pair. Then $\mathscr{H}(M, N)$ is perfect for $\operatorname{dim} N>0$.

Proof. Let $f \in \mathscr{H}(M, N)$. From the relative version of Corollary 1.3 of [E-K], we have $f=f_{k} \circ f_{k-1} \circ \cdots \circ f_{1}$, where each $f_{i}$ is supported either in an open rectangle $U_{i}$ with $U_{\imath} \cap N=\emptyset$ or in an open rectangle $U_{\imath}$ with $U_{\imath} \cap N \neq \emptyset$.

Hence we can assume that either $f_{i} \in \mathscr{H}\left(\mathbb{R}^{n}\right)$ or $f_{t} \in \mathscr{H}\left(\mathbf{R}^{n}, \mathbf{R}^{p}\right)$.

From the theorem of Mather $[\mathrm{Ma}]$ and Corollary 2.3, we have that $f$ is in the commutator subgroup of $\mathscr{H}(M, N)$. Thus $\mathscr{H}(M, N)$ is perfect. This completes the proof.

Corollary 3.2. Let $M$ be a topological manifold with boundary $\partial M$. Then $\mathscr{H}(M, \partial M)$ is perfect for $\operatorname{dim} M>1$.

Let $\mathscr{H}(L \times \mathbb{R}$, rel $L \times\{-\infty\})$ be the group of homeomorphisms of $L \times \mathbb{R}$ which are the identity on a neighborhood of $L \times\{-\infty\}$ and are isotopic to the identity, where $L$ is a closed manifold. As an immediate consequence of Theorem 1.2 of D. Mcduff $[\mathrm{Mc}]$, we have the following.

Proposition 3.3. $\quad \mathscr{H}(L \times \mathbb{R}$, rel $L \times\{-\infty\})$ is perfect.

Proposition 3.4. $\quad \mathscr{H}\left(\mathbb{R}^{n}, 0\right)$ is perfect.

Proof. Let $f \in \mathscr{H}\left(\mathbf{R}^{n}, 0\right)$. We denote by the same latter $f$ the restriction of $f$ to $\mathbb{R}^{n}-\{0\}$. Since $\mathbf{R}^{n}-\{0\}$ is homeomorphic to the product $S^{n-1} \times \mathbb{R}$, we can naturally regard that $f$ is in $\mathscr{H}\left(S^{n-1} \times \mathbb{R}\right.$, rel $\left.S^{n-1} \times\{-\infty\}\right)$.

From Proposition 3.3 , there exist $g_{\imath}, h_{\imath}$ in $\mathscr{H}\left(S^{n-1} \times \mathbf{R}\right.$, rel $\left.S^{n-1} \times\{-\infty\}\right)$ $(i=1,2, \ldots, k)$ such that $f=\Pi_{i=1}^{k}\left[g_{i}, h_{\imath}\right]$.

Since $\mathbf{R}^{n}$ is homeomorphic to the one point compactification of $S^{n-1} \times \mathbf{R}$, $S^{n-1} \times \mathbf{R} / S^{n-1} \times\{\infty\}$, we see that $g_{i}$ and $h_{i}: S^{n-1} \times \mathbf{R} \rightarrow S^{n-1} \times \mathbf{R}(i=1,2, \ldots, k)$ can be extended to homeomorphisms of $\mathbf{R}^{n}$ with compact support by mapping the 
origin to the origin.

Thus $f$ is in the commutator subgroup of $\mathscr{H}\left(\mathbb{R}^{n}, 0\right)$. This completes the proof.

Remark 3.5. Let $\operatorname{Diff} f^{\infty}\left(\mathbb{R}^{n}, 0\right)$ be the group of $C^{\infty}$-orientation preserving diffeomorphisms of $\left(\mathbb{R}^{n}, 0\right)$ isotopic to the identity. Then Theorem 1 of [F1] and Corollary 1.3 of $[\mathrm{Mc}]$ imply that $H_{1}\left(\right.$ Diff $\left.^{\infty}\left(\mathbb{R}^{n}, 0\right)\right) \cong \mathbb{R}$.

Theorem 3.6. Let $N$ be a zero dimensional submanifold of $M$. Then $\mathscr{H}(M, N)$ is perfect.

Proof. Let $f \in \mathscr{H}(M, N)$. From the relative version of Corollary 1.3 of [E-K], we have $f=f_{k} \circ f_{k-1} \circ \cdots \circ f_{1}$, where each $f_{1}$ is supported either in an open rectangle $L_{2}$ with $U_{1} \cap N=\emptyset$ or in an open rectangle $U_{t}$ with $U_{t} \cap N=$ one point .

Hence we can assume that either $f_{i} \in \mathscr{H}\left(\mathbb{R}^{n}\right)$ or $f_{1} \in \mathscr{H}\left(\mathbb{R}^{n}, 0\right)$.

From the theorem of Mather [Ma] and Proposition 3.4, we have that $f$ is in the commutator subgroup of $\mathscr{H}(M, N)$. Thus $\mathscr{H}(M, N)$ is perfect. This completes the proof.

The following is a corollary of Theorem 3.6.

Corollary 3.7 (cf. Lemma 4.4 of $[\mathrm{F}-\mathrm{I}]$ ). Let $\mathscr{H}([0,1])$ be the group of orientation preserving homeomorphisms of the interval $[0,1]$. Then $\mathscr{H}([0,1])$ is perfect.

\section{\$4. Commutators of $F(M, \mathscr{F})$, Case of Codimension Two}

Let $M$ be a compact $C^{1}$-manifold without boundary and $\mathscr{F}$ a compact Hausdorff codimension $q C^{1}$-foliation of $M$, where $\mathscr{F}$ is said to be Hausdorff if the leaf space $M / \mathscr{F}$ is Hausdorff. Then we have a nice picture of the local behavior of $\mathscr{F}$ as follows.

Proposition 4.1 ([E2]). There is a generic leaf $L_{0}$ with property that there is an open dense subset of $M$. where the leaves have all trivial holonomy and are all diffeomorphic to $L_{0}$. Given a leaf $L$, we can describe a neighborhood $U(L)$ of $L$, together with the foliation on the neighborhood as follows. There is a finite subgroup $G(L)$ of $O(q)$ such that $G(L)$ acts freely on $L_{0}$ on the right and $L_{0} / G(L)$ $\cong L$. Let $D^{q}$ be the unit disk. We foliate $L_{0} \times D^{q}$ with leaves of the form $L_{0} \times\{p t\}$. This foliation is preserved by the diagonal action of $G(L)$, defined by $g(x, y)=$ $\left(x \cdot g^{-1}, g \cdot y\right)$ for $g \in G(L), x \in L_{0}$ and $y \in D^{q}$. So we have a foliation induced on $U=L_{0} \times_{G(L)} D^{q}$. The leaf corresponding to $y=0$ is $L_{0} / G(L)$. Then there is a 
$C^{1}$-imbedding $\varphi: U \rightarrow M$ with $\varphi(U)=U(L)$, which preserves leaves and $\varphi\left(L_{0} / G(L)\right)=L$.

We consider here compact codimension two foliations. In this case, by the results of D. Epstein [E1] and R. Edwards, K. Millett and D. Sullivan [E-M-S] . we have the following: There is an upper bound on the volumes of the leaves of $\mathscr{F}$. So every compact codimension two $C^{1}$-foliation is Hausdorff.

Since $G(L)$ is a finite subgroup of $O(2), G(L)$ is either a group of $k$ rotations which is isomorphic to $\mathbb{Z}_{k}$ or a group of $l$ rotations and $l$ reflections which is isomorphic to $\mathbb{D}_{l}=\left\{\imath, v, v^{l}=v^{2}=(u v)^{2}=1\right\}$. We regard $\mathbb{Z}_{k}$ as a fixed group of rotations of $D^{2}$. Note that $\mathbb{D}_{1}$ which is generated by one reflection is isomorphic to $\mathbb{Z}_{2}$ but it is different from $\mathbb{Z}_{2}$.

Definition 4.2. A leaf $L$ is singular if $G(L)$ is not trivial. The order of $G(L)$ is called the order of holonomy of $L$. Such an $L$ is called a rotation leaf, a reflection leaf or a dihedral leaf according to whether $G(L)$ is isomorphic to $\mathbb{Z}_{k}$ $(k>1), \mathbb{D}_{1}$ or $\mathbb{D}_{l}(l>1)$.

From now on we assume that $\mathscr{F}$ has no dihedral leaves. From Proposition 4.1. there are finitely many rotation leaves in $\mathscr{F}$ because of the compactness of $M$. Let $S$ be the union of all reflection leaves of $\mathscr{F}$ and $L_{1}, \ldots, L_{r}$ all rotation leaves of $\mathscr{F}$. We denote by $B$ the leaf space $M / \mathscr{F}$ which is a compact $V$-manifold of dimension two and the quotient map $p: M \rightarrow B$ is a $V$-bundle (see I. Satake [S] for definitions). $B$ is also a topological manifold. Put $a_{\imath}=p\left(L_{1}\right)$ $(i=1,2, \ldots, r)$ and $\bar{S}=p(S) . \quad \bar{S}$ is the boundary of $B$ if $S$ is non-empty. Then we note that $p: M-S \cup L_{1} \cup \cdots \cup L_{r} \rightarrow B-\bar{S} \cup\left\{a_{1}, \ldots, a_{r}\right\}$ is a fibration with generic leaf $L$ as fibre.

Theorem 4.3. Let $M$ be a compact $C^{1}$-manifold uithout boundary and $\mathscr{F}$ a compact codimension two $C^{1}$-foliution of $M$. We assume that $\mathscr{F}$ has no dihedral leaves. Then $F(M, \mathscr{F})$ is perfect.

Proof. Every foliation preserving homeomorphism $f: M \rightarrow M$ induces a homeomorphism $\bar{f}$ of the leaf space $B$ such that the diagram commutes

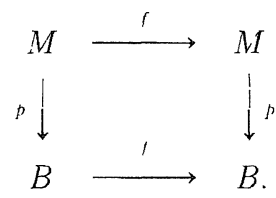

Let $L_{1}, \ldots, L_{r}$ be all rotation leaves of $\mathscr{F}$ and $a_{\imath}=p\left(L_{\imath}\right)(i=1,2, \ldots, r)$. In this case we have the natural homomorphism $\pi: F(M, \mathscr{F}) \rightarrow \mathscr{H}\left(B, \bar{S} \cup\left\{a_{1}, \ldots, a_{r}\right\}\right)$ 
defined by $\pi(f)=\bar{f}$.

Let $f \in \mathscr{H}\left(B, \bar{S} \cup\left\{a_{1}, \ldots, a_{r}\right\}\right)$. From the relative version of Corollary 1.3 of [E-K], we have $\bar{f}=\bar{f}_{k} \circ \bar{f}_{k-1} \circ \cdots \circ \bar{f}_{1}$, where each $\bar{f}_{i}$ is supported either in a small open rectangle $U_{i}$ with $U_{i} \cap\left(\bar{S} \cup\left\{a_{1}, \ldots, a_{r}\right\}\right)=\emptyset$ or in a small open rectangle $U_{i}$ with $U_{\imath} \cap \bar{S} \neq \emptyset$ and $U_{\imath} \cap\left\{a_{1}, \ldots, a_{r}\right\}=\emptyset$ or in a small open rectangle $U_{i}$ with $U_{\imath} \cap \bar{S}=\emptyset$ and $U_{\imath} \cap\left\{a_{1}, \ldots, a_{r}\right\}=$ \{one point $\}$. Thus each $\bar{f}_{\imath}$ can be lifted to $f_{\imath}$ in $F(M, \mathscr{F})$. Hence $\pi$ is onto.

Let $f \in F(M, \mathscr{F})$. We may assume that $f$ is close to the identity. It is clear that $\pi(f)=\bar{f}\left(\in \mathscr{H}\left(B, \bar{S} \cup\left\{a_{1}, \ldots, a_{r}\right\}\right)\right)$ is also close to the identity. From Corollary 3.2 and Theorem 3.6, $\mathscr{H}\left(B, \bar{S} \cup\left\{a_{1}, \ldots, a_{r}\right\}\right)$ is perfect. Thus $\bar{f}$ is in the commutator subgroup of $\mathscr{H}\left(B, \bar{S} \cup\left\{a_{1}, \ldots, a_{r}\right\}\right)$, that is, $\bar{f}=\prod_{i=1}^{s}\left[\bar{g}_{i}, \bar{h}_{t}\right]\left(\bar{g}_{t}, \bar{h}_{t} \in\right.$ $\left.\mathscr{H}\left(B, \bar{S} \cup\left\{a_{1}, \ldots . a_{r}\right\}\right)\right)$. Here $\bar{g}_{\imath}$ and $\bar{h}_{t}(i=1,2, \ldots, k)$ can be supported in small neighborhoods in $B$. By lifting $\bar{g}_{\imath}$ and $\bar{h}_{\imath}$ to $g_{\imath}$ and $h_{\imath}$ in $F(M, \mathscr{F})$, we have $f^{\prime}=$ $\prod_{i=1}^{s}\left[g_{l}, h_{t}\right]$. Since $\pi\left(f \circ\left(f^{\prime}\right)^{-1}\right)=i d, f \circ\left(f^{\prime}\right)^{-1}$ is contained in $L(M, \mathscr{F})$, where $L(M, \mathscr{F})$ is the group of leaf preserving homeomorphisms of $(M, \mathscr{F})$ which are isotopic to the identity. From Theorem 3.2 of $[\mathrm{F}-\mathrm{I}], f \circ\left(f^{\prime}\right)^{-1}$ is in the commutator subgroup of $L(M, \mathscr{F})$. Hence $f$ is in the commutator subgroup of $F(M, \mathscr{F})$. Thus $F(M, \mathscr{F})$ is perfect. This completes the proof.

Corollary 4.4. Let $M$ be a compact $C^{1}$-manifold without boundary and F a compact codimension two $C^{\mathbf{l}}$-foliation of $M$. We assume that $\pi_{1}(L) \cong \mathbb{Z}$ for every leaf $L$ of $\mathscr{F}$. Then $F(M, \mathscr{F})$ is perfect.

Proof. Take a singular leaf $L$ of $\mathscr{F}$. Then $G(L)$ is isomorphic to a finite cyclic group since $\pi_{1}(L) \cong Z$ for every leaf $L$ of $\mathscr{F}$. Thus any dihedral leaves do not appear in $\mathscr{F}$ and hence the corollary follows from Theorem 4.3.

Corollary 4.5. Let $M$ be a compact 4-dimensional $C^{1}$-manifold without boundary and $\mathscr{F}$ a $C^{1}$-foliation of $M$ by orientable surfaces. If the genus of a generic leaf is even, then $F(M, \mathscr{F})$ is perfect.

Proof. Take a generic leaf $L_{0}$ and a singular leaf $L$ of $\mathscr{F}_{F}$. Then we have a regular covering $\pi: L_{0} \rightarrow L$ with structure group $G(L)$. Thus if the genus of $L_{0}$ is even, then the order of $G(L)$ is not even, hence $G(L)$ can not be a dihedral group. Hence the corollary follows from Theorem 4.3.

\section{§5. Commutators of $F(M, F)$, Case of Codimension Three}

In this section we consider the group of foliation preserving homeomorphisms for compact Hausdorff codimension three foliations.

Let $\mathscr{F}$ be a compact Hausdorff codimension three $C^{1}$-foliation of a compact 
manifold $M$. We assume that $\pi_{1}(L) \cong \mathbb{Z}$ for every leaf $L$ of $\mathscr{F}$. Then $G(L)$ in Proposition 4.1 is isomorphic to a finite cyclic subgroup of $O(3)$. Then we have the following.

Proposition 5.1 (cf. [F2]). Each cyclic subgroup $G$ of $O(3)$ is classified as follows:

(i) Type $I(G \subset S O(3))$ : $G$ is isomorphic to $\mathbb{Z} / n \mathbb{Z}$ which is generated by

$$
A=\left(\begin{array}{ccc}
\cos 2 \pi / n & -\sin 2 \pi / n & 0 \\
\sin 2 \pi / n & \cos 2 \pi / n & 0 \\
0 & 0 & 1
\end{array}\right) .
$$

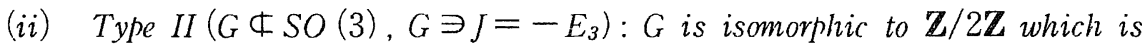
generated by $J$.

(iii) Type III (GథSO (3), G尹J): G is isomorphic to $\mathbb{Z} / n \mathbb{Z}$ (n: even) which is generated by $J A$.

Definition 5.2. We say such a singular leaf $L$ is of type $I$, type II type $\mathbb{I I}_{1}$ or type $\mathbb{I I}_{2}$ if $G(L)$ is of Type $I$, Type II. Type III and $n=2$, or Type III and $n \geq 4$.

We can clarify the local structure of the leaf space $M / \mathscr{F}$ using Proposition 5.1 as follows.

Let $U(L)$ be a saturated neighborhood of $L$ as in Proposition 4.1.

(1) In case $L$ is of type $\mathbb{I}, L^{T}(L) / \mathscr{F}$ is homeomorphic to $C \times(-1,1)$, where $C$ is the quotient space of $D^{2}$ by a linear action of $\mathbb{Z} / n \mathbb{Z}(\subset S O(2))$. The union of leaves of type $I$ corresponds to $\{(0,0)\} \times(-1,1)$. Therefore $U(L) / \mathscr{F}$ is still a topological manifold.

(2) In case $L$ is of type $I I, L^{T}(L) / \mathscr{F}$ is homeomorphic to the suspension of the projective plane $P^{2}$.

(3) In case $L$ is of type $\mathbb{I I}_{1}, U(L) / \mathscr{F}$ is homeomorphic to $D^{2} \times[0,1)$. The union of leaves of type $\mathrm{III}_{1}$ corresponds to $D^{2} \times\{0\}$. Therefore the leaves of type $\mathrm{III}_{1}$ correspond to the points of the boundary of $M / \mathscr{F}$.

(4) In case $L$ is of type $\mathbb{I}_{2}, U(L) / \mathscr{F}$ is homeomorphic to $C \times[0,1)$, where $C$ is that in (1). The point $\{(0,0)\} \times\{0\}$ corresponds to the leaf of type $\mathbb{I}_{2}$, the points in $\{(0,0)\} \times(0,1)$ correspond to the leaves of type $I$ and the points in $(C-(0,0)) \times\{0\}$ correspond to the leaves of type $\mathbb{I l}_{1}$.

In case $L$ is of type II . $U(L) / \mathscr{F}$ is homeomorphic to the one point compactification of the product $P^{2} \times \mathbf{R}, P^{2} \times \mathbf{R} / P^{2} \times\{\infty\}$, which is denoted by $S\left(P^{2}\right) . S\left(P^{2}\right)$ is not a manifold but has a manifold structure except for the infinity point $P^{2} \times\{\infty\}=\infty$. 
Let $\mathscr{H}\left(S\left(P^{2}\right), \infty\right)$ be the group of homeomorphisms of $S\left(P^{2}\right)$ which are isotopic to the identity by an isotopy fixed outside a compact set and leave $\infty$ fixed.

Proposition 5.3. $\quad \mathscr{H}\left(S\left(P^{2}\right), \infty\right)$ is perfect.

Proof. Let $f \in \mathscr{H}\left(S\left(P^{2}\right), \infty\right)$. We denote by the same letter $f$ the restriction of $f$ to $P^{2} \times \mathbb{R}$. By Proposition 3.3, we have

$$
f=\prod_{i=1}^{k}\left[g_{i}, h_{\imath}\right], g_{i}, h_{t} \in \mathscr{H}\left(P^{2} \times \mathbb{R}, \text { rel } P^{2} \times\{-\infty\}\right)(i=1,2, \ldots, k) .
$$

Then we see that $g_{1}$ and $h_{1}: P^{2} \times \mathbb{R} \rightarrow P^{2} \times \mathbb{R}(i=1,2, \ldots, k)$ can be extended to homeomorphisms of $S\left(P^{2}\right)$ with compact support by mapping the infinity point to the infinity point.

Thus $f$ is in the commutator subgroup of $\mathscr{H}\left(S\left(P^{2}\right), \infty\right)$. This completes the proof.

Theorem 5.41. Let $M$ be a compact $C^{1}$-manifold without boundary and F a compact Hausdorff codimension three $C^{1}$-foliation of $M$ with leaf space $B$. We assume that $\pi_{1}(L) \cong \mathbb{Z}$ for every leaf $L$ of $\mathscr{F}$ and $\mathscr{F}$ has no leaves of type $\mathbb{I I I}_{2}$. Then $F(M, \mathscr{F})$ is perfect.

Proof. From the assumption, $B$ has a manifold structure except for points corresponding to leaves of type II. Since $M$ is compact, such points are finite. We denote them by $a_{1}, \ldots, a_{r}$. Let $N$ be the submanifold of $B$ corresponding to the union of leaves of type $I$ and type $I_{1}$. Then we have the natural homomorphism $\pi: F(M, \mathscr{F}) \rightarrow \mathscr{H}\left(B, N \cup\left\{a_{1}, \ldots, a_{r}\right\}\right)$ defined by $\pi(f)=$ $\bar{f}$ as in the proof of Theorem 4.3.

Let $\bar{f} \in \mathscr{H}\left(B, N \cup\left\{a_{1}, \ldots, a_{r}\right\}\right)$. From the relative version of Corollary 1.3 of [E-K], we have $\bar{f}=\bar{f}_{k} \circ \bar{f}_{k-1} \circ \cdots \circ \bar{f}_{1}$, where each $\bar{f}_{\imath}$ is supported either in a small open neighborhood $L_{t}$, with $L_{t}^{r} \cap\left(N \cup\left\{a_{1, \ldots, a_{r}}\right\}\right)=\varnothing$ or in a small open neighborhood $U_{2}$ with $U_{1} \cap N \neq \emptyset$ and $U_{2} \cap\left\{a_{1}, \ldots, a_{r}\right\}=\emptyset$ or in a small open neighborhood $U_{t}$ with $L_{i} \cap N=\varnothing$ and $L_{i} \cap\left\{a_{1}, \ldots, a_{r}\right\}=$ \{one point $\}$. Thus each $\bar{f}_{i}$ can be lifted to $f_{1}$ in $F(M, \mathscr{F})$. Hence $\pi$ is onto.

Let $f \in F(M, \mathscr{F})$. We may assume that $f$ is close to the identity. It is clear that $\pi(f)=\bar{f}\left(\in \mathscr{H}\left(B, N \cup\left\{a_{1}, \ldots, a_{r}\right\}\right)\right)$ is also close to the identity. From Theorem 3.1 and Proposition 5.3. $\mathscr{H}\left(B, N \cup\left\{a_{1}, \ldots, a_{r}\right\}\right)$ is perfect. Thus $\bar{f}$ is in the commutator subgroup of $\mathscr{H}\left(B, N \cup\left\{a_{1}, \ldots, a_{r}\right\}\right)$, that is, $\bar{f}=\prod_{i=1}^{s}\left[\bar{g}_{l}, \bar{h}\right]\left(\bar{g}_{l}, \bar{h}_{l} \in\right.$ $\left.\mathscr{H}\left(B, N \cup\left\{a_{1}, \ldots, a_{r}\right\}\right)\right)$. Here $\bar{g}_{\imath}$ and $\bar{h}_{\imath}(i=1,2, \ldots, k)$ can be supported in small neighborhoods in $B$. By lifting $\bar{g}_{\imath}$ and $\bar{h}_{\imath}$ to $g_{\imath}$ and $h_{\imath}$ in $F(M, \mathscr{F})$, we have $f^{\prime}=$ $\prod_{l=1}^{s}\left[g_{l}, h_{l}\right]$. Since $\pi\left(f \circ\left(f^{\prime}\right)^{-1}\right)=i d, f \circ\left(f^{\prime}\right)^{-1}$ is contained in $L(M, \mathscr{F})$. From Theorem 3.2 of $[\mathrm{F}-\mathrm{I}] . f \circ\left(f^{\prime}\right)^{-1}$ is in the commutator subgroup of $L(M, \mathscr{F})$. 
Hence $f$ is in the commutator subgroup of $F(M, \mathscr{F})$. Thus $F(M, \mathscr{F})$ is perfect. This completes the proof.

\section{References}

[E-K] Edwards, R. D., Kirby. R. C., Deformations of spaces of imbeddings, Amn. of Math., 93 (1971), 63-88.

[E-M-S] Edwards, R., Millett, K.. Sullivan, D., Foliations with all leaves compact, Topology, 16 (1977), 13-32.

[E1] Epstein, D.B.A., Periodic flows on three-manifolds, Ann. of Math., 95 (1976), 68-82.

[E2] - Foliations with all leaves compact. Ann. Inst. Fourier, Grenoble, 26 (1976), 265-282.

[F1] Fukui, K., Homologies of the group Diff $\left(\boldsymbol{R}^{n}, 0\right)$ and its subgroups, J. Math. Kyoto Lnvv., 20 (1980), 475-487.

[F2] - Stability of Hausdorff foliations of 4-manifolds by circles, Math. Japon., 34-6 (1989), 925-932.

[F-I] Fukui, K., Imanish1, H., On commutators of foliation preserving homeomorphisms, to appear in J. Math. Soc. Japan.

[Ma] Mather, J. N., The vanishing of the homology of certain groups of homeomorphisms. Topology, 10 (1971), 297-298

[Mc] McDuff, D., The lattice of normal subgroups of the group of diffeomorphisms or homeomorphisms of an open manifold, J. London Math. Soc., 18-2 (1978), 353-364.

[S] Satake, I., The Gauss-Bonnet theorem for V-manifolds, J. Math. Soc. Japan, 9-4 (1957). 464-492. 
